\title{
BMJ Open MAGDALENA: study protocol of a randomised, placebo-controlled trial on cognitive development at 2 years of age in children exposed to SSRI in utero
}

To cite: Heinonen $\mathrm{E}$,

Szymanska-von Schultz B, Kaldo V, et al. MAGDALENA: study protocol of a randomised, placebo-controlled trial on cognitive development at 2 years of age in children exposed to SSRI in utero. BMJ Open 2018;8:e023281. doi:10.1136/ bmjopen-2018-023281

- Prepublication history and additional material for this paper are available online. To view these files, please visit the journal online (http://dx.doi. org/10.1136/bmjopen-2018023281).

Received 29 March 2018 Revised 12 June 2018 Accepted 20 June 2018

Check for updates

(C) Author(s) (or their employer(s)) 2018. Re-use permitted under CC BY-NC. No commercial re-use. See rights and permissions. Published by BMJ.

For numbered affiliations see end of article.

Correspondence to

Mrs. Essi Heinonen; essi.heinonen@ki.se

\section{ABSTRACT}

Introduction Ten per cent of all pregnant women are depressed. Standard therapy of pregnant women with moderate depression is selective serotonin reuptakeinhibitors (SSRI). Observational studies on neurodevelopment after fetal SSRI exposure show conflicting results. Our primary objective is to compare the cognitive development in children exposed to sertraline and maternal depression with those exposed to maternal depression and placebo in utero. We hypothesise that there is a significant neurodevelopmental difference between the groups. As a secondary objective, we study the add-on effect of sertraline to internet-based cognitive behavioural therapy (ICBT) to treat moderate depression during pregnancy.

Methods and analysis MAGDALENA is a randomised, placebo-controlled, double-blinded trial in Stockholm Healthcare Region with 2.3 million inhabitants. The women are recruited in weeks 9-21 of pregnancy either through Antenatal Health Clinics or through social media. They are to be diagnosed with moderate depression without ongoing antidepressive therapy or any serious comorbidity. The women in the intervention arm receive sertraline combined with a 12-week period of ICBT; the control arm is treated with placebo and ICBT. We assess the cognitive development in the offspring at the age of 2 years using Bayley Scales of Infant and Toddler Development, third edition (BSID-III). We aim at recruiting 200 women, 100 women in each treatment arm, to ensure statistical power to detect a clinically relevant difference between the groups.

Ethics and dissemination This randomised trial will provide long-sought evidence about the effects of SSRI and maternal depression during pregnancy on the neurodevelopment in the offspring. The study is approved by the Regional Ethical Review Board at Karolinska Institutet in Stockholm and the Swedish Medical Products Agency. It is registered with the European Clinical Trials Database (EudraCT), Number: 2013-004444-31. Results will be disseminated at scientific conferences, published in peer-reviewed journals and made available to the public.
Strengths and limitations of this study

- To our knowledge, this is the first randomised controlled trial to separate the neurodevelopmental effects of fetal selective serotonin reuptake inhibitor exposure from the possible effects from the underlying maternal depression. We consider that a randomised controlled trial is the most valid method to find answers to this complex question.

- The highly structured and homogenous nature of the internet-based cognitive-behavioural therapy (ICBT) given to both groups reduces the risk of confounding by therapist factors and should make the add-on effects of sertraline clear-cut.

- The ICBT protocol is in Swedish only and cannot be performed with an interpreter, which requires Swedish literacy. Therefore, the generalisability of our study results is reduced, and the recruitment potential is limited in multicultural areas.

- The severity of depression is monitored with Montgomery-Åsberg Depression Scale, which contains items that can be related both to depression and to pregnancy, which complicates the interpretation of the results.

- Due to the evaluation at 2 years of age, we cannot answer the question about autism since it is not easily diagnosed at this early age. We plan to follow up our cohort long term.

Trial registration number EudraCT2013-004444-31; Pre-results.

\section{INTRODUCTION}

\section{Background}

Although approximately $10 \%$ of pregnant women are depressed, the prescription rate of antidepressants decreases during pregnancy as compared with the preceding months. ${ }^{1-3}$ Untreated prenatal depression is associated 
with pregnancy complications such as pre-eclampsia, ${ }^{4}$ low birth weight and prematurity ${ }^{5}$ and increased plasma levels of stress hormones in the pregnant woman and the neonate. ${ }^{67}$ Published data show that $2.4 \%$ of pregnant women in Sweden during years $2006-20128^{8}$ and $6 \%$ of the ones in USA during the years $2001-2013^{9}$ were treated with selective serotonin reuptake inhibitor (SSRIs). Our own unpublished data of prescriptions for Stockholm Healthcare Region extracted from the Swedish Drug Registry show that this percentage is constant at $5 \%$ in pregnant women in Stockholm during the period 20142016. Increased risk for SSRI-related birth defects has not been reported for most SSRIs except that fluoxetine and paroxetine seem to be associated with a small increase in the risk for heart defects. ${ }^{10}$

The question if fetal SSRI exposure causes long-term neurodevelopmental effects remains unanswered. ${ }^{11}$ Observational studies have reported a twofold increased risk for autism spectrum disorder, ${ }^{12-15}$ but recently two large register studies explained this increase by confounding factors, emphasising the role of genetic and social factors as opposed to drug exposure alone. ${ }^{16}{ }^{17} \mathrm{~A}$ recent review also concluded that the increase was not significant when comparing with women with previous affective disease or in sibling studies. ${ }^{18}$ To our knowledge, there is no previous randomised controlled trial in this field.

Our main objective is to clarify the cognitive effects on the children by pursuing a randomised, controlled, double-blind study. We compare the cognitive development at 2 years of age in children with women with moderate depression during pregnancy, treated with either internet-based cognitive-behavioural therapy (ICBT) and sertraline (an SSRI compound) or ICBT and placebo. The Swedish acronym of the study, MAGDALENA, reflects this main objective: 'Maternal Affective Disease during Pregnancy: Depression and Antidepressant Drugs and Effects on the Neurological Development and Adaptation'.

Our secondary objective is to understand the add-on effect of sertraline to ICBT, a web-based self-treatment programme with active therapist support over the internet. ICBT is a well-documented treatment for depression used in regular psychiatric care. ${ }^{19}$ It has been shown that pregnant women feel that psychotherapy needs to take the special case of being pregnant into account to be credible and engaging. ${ }^{20}$ Postpartum depression has been targeted in online trials ${ }^{20-23}$ but not antenatal depression. Because of this, we developed an ICBT programme to be useful for treatment of antenatal depression based on the ICBT for depression used at our Internet Psychiatry Clinic. The modified ICBT was tested in a pilot RCT with good results. The effect size compared with regular maternity care was of clinical importance and significant (Hedges $g=1.21, \mathrm{p}<0.001){ }^{24}$

The Swedish guidelines state that mild and moderate depression should primarily be treated with psychotherapy or antidepressants. Cognitive-behavioural therapy and interpersonal therapy are recommended as the first choice of therapy. Severe depression should be treated with antidepressants or electroconvulsive therapy (ECT). The recommendation does not give guidance on the value of combination therapies for any level of depression. ${ }^{25}$ Combination therapy with CBT and antidepressants appear more effective than antidepressant therapy alone in treatment of major depression, ${ }^{26}$ but this has not been studied in pregnant women. Given the in utero effects of untreated depression during pregnancy, ${ }^{7}$ it might be rational with a dual treatment to achieve prompt symptom reduction. The effects of ICBT are comparable with the effects seen with traditional CBT, hence having the potential to be a cost-effective and accessible treatment alternative. ${ }^{19} 242728$ Our large group of patients and unique design with and without sertraline treatment allows us also to pursue exploratory tertiary studies in the pregnant women and in the newborns. ${ }^{29} 30$

\section{Objectives}

Primary and secondary objectives

Our primary objective is to study cognitive development at 2 years of age in children prenatally exposed to moderate maternal depression treated either with sertraline and ICBT (intervention) or with ICBT and placebo (control). Our hypothesis is that there is a significant neurodevelopmental difference between 2-year-old children exposed to the intervention treatment (sertraline) as compared with the control treatment, measured with established scoring methods. If no difference is detected, we will include a non-inferiority analysis to test if the neurodevelopment in the exposed group is at least as good as for the control group of children.

Our secondary objective is to evaluate the add-on effect of treatment with sertraline to an ICBT programme for antenatal depression. Our hypothesis is that sertraline provides a significant add-on effect to ICBT therapy.

\section{Exploratory tertiary objectives}

The large material and unique design with one SSRI-exposed and one placebo-treated arm allows exploratory studies on tertiary aspects related to physiological and adaptive changes in depressed pregnant women and in their newborns. We will study the risk of pre-eclampsia, bleeding and caesarean section and potential changes of biochemical parameters and hormones during the course of pregnancy in the two study groups. ${ }^{41-33}$ Paediatric exploratory objectives include clarification of type and prevalence of signs and symptoms of neonatal maladaptation and risk of admission to a neonatal care unit. The design allows a subgroup study of maternal-fetal attachment and bonding style differences between the groups. ${ }^{34}$

We will explore interindividual variation in sertraline pharmacokinetics during and after pregnancy and in newborns and how it might relate to different genetic variants of drug metabolising enzymes in pregnant women and newborns. ${ }^{35-37}$ Likewise we will explore the epigenetic differences between the groups in mothers and newborns. ${ }^{38}$ 


\section{METHODS AND ANALYSIS}

\section{Trial design and setting}

The MAGDALENA study is a prospective, randomised, placebo-controlled, double-blinded, single-centred, clinical investigation of pregnant women with moderate depression recruited from the Stockholm catchment area (2.3 million inhabitants and 28000 deliveries annually) with multiple delivery units. The study group receives sertraline (or placebo) clinically titrated to a maximum daily dose of $150 \mathrm{mg}$. The study and control groups are both treated with ICBT. ${ }^{24}$ The trial design includes two different recruitment pathways (A and B) (figure 1). The primary outcome is achieved with scoring of the neurodevelopment of the child at 2 years of age. The framework for neurodevelopment is primarily a superiority test. The add-on effect of SSRI to ICBT is also a superiority test. The tertiary objectives are exploratory.

\section{Recruitment and blinding}

Pregnant women are recruited either from the antenatal health clinics (figure 1, pathway A) or through social media and our study website www.magdalenastudien.se (figure 1, pathway B). Pregnant women visit their midwives at the antenatal clinics, commonly with their partners, around 10 times during pregnancy free of charge. In pathway $\mathrm{A}$, the midwives inform about the study at a regular visit. The recruitment through the study website, pathway $\mathrm{B}$, is simplified by informing about the study to potential subjects continuously through social media, pregnancy-related information platforms, different blogs and podcasts. Smartphone pregnancy applications are popular, reaching about $75 \%$ of pregnant women in cross-sectional studies in English and Spanish speaking countries. We assume similar, if not higher, usage in Sweden. ${ }^{39}{ }^{40}$ In both pathways, the women are directed our study platform at the internet psychiatry website, www. internetpsykiatri.se. They are asked to fill the Edinburgh Postnatal Depression Scale (EPDS) ${ }^{41}$ self-test to assess the degree of depressive symptoms as well as to sign the first informed consent form. Regardless of recruitment pathway, the women will continue their regular visits at their home antenatal clinic.

We have chosen 13 or more points on the EPDS for further evaluation of eligibility for the study. The reason is that it has been found to be the optimal cut-off point with $77 \%$ sensitivity and $94 \%$ specificity for detection of depressive symptoms during pregnancy. ${ }^{41}$ The subjects that score 13 points or more on the EPDS self-test and do not fill any exclusion criteria are scheduled to meet one of our three study psychiatrists for clinical evaluation, including Structured Clinical Interview for DSM IV axis I (SCID-I) directed interview (SCID-I disorders) ${ }^{42}$ review of eligibility criteria and signing of the final informed consent form (visit 1, figure 1, table 1). The included women are allocated to the next consecutive patient number and randomised to treatment by Karolinska Trial Alliance (KTA) (see below). The KTA will also ensure correct blinding for all trial participants, care providers, outcome assessors and data analysts, including labelling of the study drug packages. The drug treatment with study drug (sertraline or placebo) is initiated after visit 2 (figure 1). At the same time, the women start ICBT treatment using the internet psychiatry website. ${ }^{1924}$ Both study and control groups receive capsules of identical appearance and packing (see below). The treatment code is broken, and a new clinical evaluation is performed by the study psychiatrist 1 month after the delivery. The treatment will be continued for 1 year after the delivery for all patients in the active treatment arm. The women in the placebo group that have not reached remission will also be offered treatment with SSRI. Both the women and the psychiatrist are forbidden to inform anyone in the study about which group they have belonged to to ensure the continued blinding of the study. This is particularly important regarding the paediatricians and paediatric psychologists assessing the children.

\section{The study drugs}

Sertraline is one of the two first-line recommended drugs for depression during pregnancy according to the Drug and Therapeutics Committee in Stockholm Healthcare Region. ${ }^{4-45}$ Sertraline is also widely used across Europe for treatment of depression during pregnancy. ${ }^{3} 4647$ This is why sertraline was the preferred choice as the study drug.

The investigational medicinal product (IMP) is a capsule containing either sertraline hydrochloride or placebo manufactured by APL (Apoteket Produktion \& Laboratorier AB, Stockholm, Sweden). The capsules of active drug are made of hard gelatine and filled with sertraline hydrochloride corresponding to $25 \mathrm{mg}$ sertraline using Zoloft (25 mg sertraline film-coated tablets, Pfizer, New York, USA) and with a microcrystalline cellulose filler. The placebo capsules are made of identical microcrystalline cellulose filler embedded into an identical hard gelatine capsule. The IMP is delivered by the producer in sealed HD polyethylene containers, labelled according to a randomisation list. Patients are randomised to treatment by KTA with block randomisation. The size of these blocks is blinded for the investigators.

The treatment with sertraline (or placebo) starts with titration of a dose of one capsule $(25 \mathrm{mg}$ sertraline or placebo) once daily for 5 days, after which the dose is increased to two capsules ( $50 \mathrm{mg}$ of sertraline or placebo) once daily until the first treatment evaluation (visit 3, figure 1). At visit 3, the daily dose can be increased to four capsules ( $100 \mathrm{mg}$ of sertraline or placebo) once daily if clinically indicated, with titration of three capsules ( $75 \mathrm{mg}$ of sertraline or placebo) once daily for 5 days. At visit 4 after 13 weeks of treatment, the dose can be increased further to a maximum $150 \mathrm{mg}$ daily, without titration, if clinically needed. The decision of need to increase drug dosages is based on MADRS scores and a clinical evaluation made by the study psychiatrist. To follow and increase the adherence to treatment, the women will get a limited amount of capsules at each visit, 


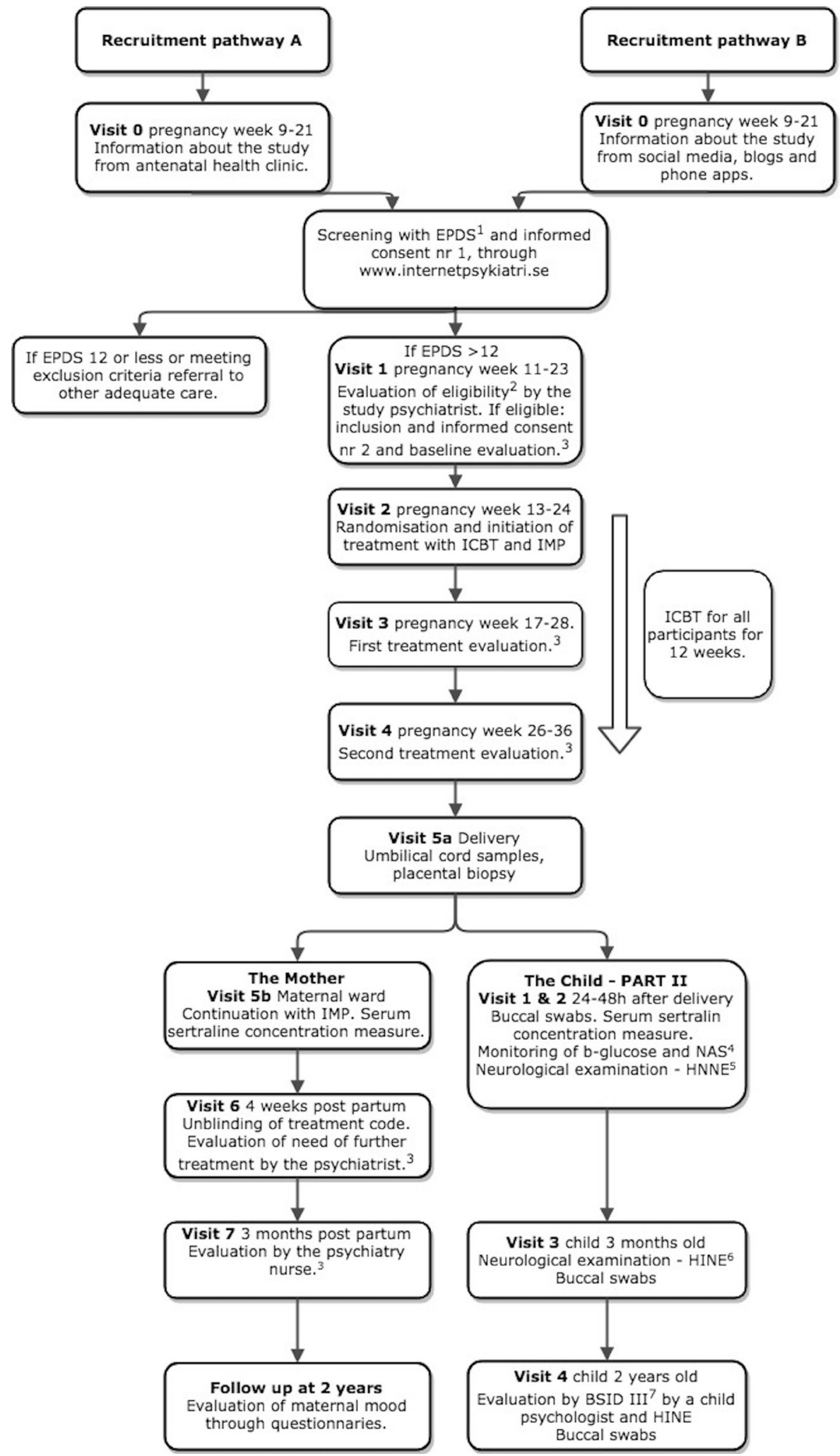

Figure 1 Trial design and participant timeline for the two recruitment pathways. The figure also shows the treatment with placebo/sertraline, the investigational medical product (IMP) from visit 2 (pregnancy weeks 13-24) to visit 6 (4 weeks postpartum) and the internet-based cognitive-behavioural therapy (ICBT) for both groups for 12 weeks, between visit 2 and visit 4 (pregnancy weeks 26-36) and monitoring of therapy for the two groups. The postpartum follow-up of both mother and child are shown. The different scales and examinations are as presented in the figure: (1) Edinburgh Postnatal Depression Scale (EPDS), ${ }^{41}$ (2) diagnosis of moderate depression is confirmed according to clinical standard evaluation. Inclusion and exclusion criteria presented in table 1. (3) Evaluation with Montgomery-Åsberg Depression Scale (MADRS), ${ }^{64}$ (4) Modified Finnegan Neonatal Abstinence Scale (NAS), ${ }^{58}$ (5) Hammersmith Neonatal Neurological Examination (HNNE), ${ }^{57}$ (6) Hammersmith Infant Neurological Examination (HINE) ${ }^{65}$ and (7) Bayley Scales of Infant and Toddler Development III (BSID III). ${ }^{48}$ 
Table 1 Inclusion and exclusion criteria for the MAGDALENA study

$\begin{array}{ll}\text { Inclustion citeria } & \text { Age }>18 \text { years. } \\ \text { Pregnant in week 9-21 after last menstrual period. } \\ \text { Verified moderate depression according to SCID-I (Structured Clinical Interview for DSM IV axis I } \\ \text { disorders), with or without a concomitant anxiety disorder, and a clinical evaluation. } \\ \text { Ability to use the internet platform for ICBT in Swedish as assessed by the study midwife. } \\ \text { Reported ability to participate in all study visits for mother and child. } \\ \text { Reported abuse of alcohol or drugs. } \\ \text { Reported serious psychiatric disorder.* } \\ \text { Known allergy or idiosyncratic reaction to sertraline. } \\ \text { Ongoing medication with antidepressants, mood stabilisers, central stimulants, antiepileptic drugs, } \\ \text { opiates, insulin, oral antidiabetics, antiarrhythmics or steroids. } \\ \text { A severe somatic disease† that requires medical treatment. } \\ \text { A high suicidal risk during screening or when included into the study. These women will be } \\ \text { excluded from the study and actively transferred to necessary psychiatric care. }\end{array}$

*Psychosis, bipolar disorder, severe melancholic or psychotic depression, severe personality disorder, autism, mental retardation or attention deficit and hyperactivity disorder (ADHD)/attention deficit disorder (ADD) with ongoing drug treatment or contact with specialist psychiatry clinic.

†Severe heart and lung disease, kidney disease, liver disease, diabetes mellitus, epilepsy with drug treatment and any severe somatic disease that requires regular treatment with systemic steroids.

motivating them to return for the next visit and giving the study midwife a way to notice if the subject is not taking the drug. Additional telephone contacts with the psychiatry nurse are performed if concerns about the treatment effect are raised, and an additional visit to the psychiatrist is scheduled if necessary. The study protocol attached as an online supplementary appendix gives a detailed description of the treatments, monitoring of mothers and follow-up of both mothers and infants.

\section{The ICBT}

The ICBT treatment is 12 weeks long and guided by a psychologist who is available online. It consists of an online platform where patients log in to answer questionnaires and work with self-help material covering the core materials in CBT for depression including interactive worksheets and exercises. They are also assigned weekly homework to report to their personal therapist. A direct messaging system allows for asynchronous correspondence with one of the therapists at any time and get a reply within a day or two. The platform allows monitoring to what extent the patients adhere to the therapeutic sessions.

The pregnancy-related adaptations of the ICBT treatment consist of an extra module on pregnancy-related symptoms that can lower mood at the beginning of the treatment and an extra module on relationships at the end of treatment. Other than that, the interventions mostly mirror those described and used by us in our ICBT-platform for non-pregnant adults. ${ }^{19}$ The examples and cases included in the treatment concern pregnant women and their thoughts on pregnancy and becoming a parent and expanding their family. The core methods of the treatment are behavioural activation (increasing positive and valued activities) and cognitive restructuring (challenging own thoughts and distance oneself from dysfunctional thoughts and rumination).

\section{Outcome measurements}

Primary outcome: effect on neurodevelopment at 2 years of age The cognitive development at 2 years of age is the primary outcome. The outcome in children exposed and not exposed to sertraline in utero is measured with the standardised Bayley Scales of Infant and Toddler Development III (BSID-III $)^{48}$ and compared between the groups. The BSID-III consists of the Cognitive, Language and Motor Scales that are performed by a child psychologist, and the Social Emotional and the Adaptive Scales that are parental questionnaires. The Cognitive and Language scales of the BSID-III have proven to be good predictors of preschool mental test performance. ${ }^{49}$ These scales are largely used in screening for cognitive developmental delays ${ }^{50}$ and have been used in studies of cognitive development after intrauterine drug exposure. ${ }^{51-53}$ Results from the subscales will be converted to composite scores with a median of 100 and an SD of 15 . For each subscale, a composite score of $<85(<-1 \mathrm{SD})$ will be considered abnormal. A difference of 7 points $(0.5 \mathrm{SD})$ between the groups will be considered significant, which is in line with the assumptions made in previous similar studies. ${ }^{50}$

\section{Sample size calculation}

A difference of 7 points (SD 0.5) in the results of BSID-III at 2 years of age is considered a minimally important clinical difference based on the previous follow-up studies performed with BSID. ${ }^{505} 54$ We calculated that, with a power of $80 \%(\alpha: 0.05)$, this would show a significant difference in a sample of 73 participating children in each group. We hypothesise that there is a significant difference between the groups and have performed a calculation for a superiority test (effect on BSID for the sertraline exposed study group). We aim at recruiting 100 mothers in each group to rely on a result that has a margin for loss to follow-up, considering the longterm nature of this study. If no difference is found our 
study design will also provide enough power to test the non-inferiority hypothesis. According to a non-inferiority power calculation method,$^{55}$ we will need 50 patients in each group to have power of $80 \%$ ( $\alpha: 0.05$ ) to confirm that the SSRI-exposed children lies within a tolerance margin of 7 points below the control children. This corresponds to an effect size of 0.5 (Cohen's $d$ ). As a more conservative sensitivity test for non-inferiority, we will also use a tolerance margin of 5 points, which would correspond to an effect of $d=0.36$. This requires 97 patients in each group.

\section{Secondary outcome}

The main psychiatric secondary outcome is the add-on effect of sertraline to the treatment with ICBT on depressive symptoms measured with Montgomery-Åsberg Depression Rating Scale, Self-Report (MADRS-S) ${ }^{56}$ which is performed weekly throughout the 12 week treatment period.

\section{Sample size calculation for the secondary outcome}

The additional effect of sertraline as compared with standard ICBT plus placebo will be evaluated by comparing effects on MADRS-S scores between the groups. Data on the total effect of CBT combined with antidepressant therapy is limited. The standardised effect of psychotherapy can be as high as 0.74 when compared with placebo and 0.38 when compared with face-to-face psychotherapy. ${ }^{26}$ ICBT is largely self-directed even with therapist guidance, which is likely to put a higher demand on the patients in terms of initiative and maintaining the motivation compared with face-to-face-treatment. Therefore, it is likely that the added effect of SSRI will be larger than what has been found in face-to-face CBT, though not as large as when compared with placebo alone. We therefore hypothesise a standardised effect size of $d=0.50$ (Cohen). This is also the minimal added effect that we would consider clinically relevant considering the possibility of negative side effects and the documented efficacy of ICBT alone. ${ }^{24} \mathrm{~A}$ sample size calculation with standardised effect equal to 0.5 (Cohen), alfa-value $=0.05$ and power $80 \%$ requires 64 persons in each group. Therefore, to recruit 100 patients in each group will fulfil the power calculation.

\section{Exploratory tertiary studies}

Exploratory maternal outcomes include the effects on levels on prolactine and cytokines and the risk for postpartum bleeding measured in millilitres and postpartum anaemia measured with haemoglobin day 2 for the SSRI-exposed women as compared with the placebo-exposed women. We also evaluate the risk of pre-eclampsia, placental abruption and increased caesarean section rate.

The neonatal exploratory outcomes include admission to neonatal care, neurological evaluation with Hammersmith Neonatal Neurological Examination ${ }^{57}$ and modified Finnegan Neonatal Abstinence Scales ${ }^{58}$ and measurement of glucose and concentrations of sertraline in plasma.
In a subcohort, blood samples from the umbilical cord and placental biopsies are taken to study epigenetic effects (DNA methylation, genome width, and gene and mRNA expression) between SSRI exposed and the non-exposed within this cohort.

We will study differences in maternal-child bonding/ attachment during and after birth and also breastfeeding occurrence. Prenatal Attachment Inventory-Revised questionnaires and video recordings analysed by 'Dr Feldman's micro-coded behaviours' will be used, which is a valid tool to study attachment ${ }^{59} \mathrm{~A}$ comparison will be made between the two groups.

The pharmacokinetic analyses include studies of interindividual variability in plasma sertraline concentrations and its change during the course of pregnancy and postpartum (mean concentration/ (dose in $\mathrm{mg} / \mathrm{kg}$ ) with CIs. Pharmacogenetic variants of drug metabolising enzymes and transporters will be studied in pregnant women and newborns. Umbilical cord blood and venous blood from the neonate will be taken, and plasma sertraline levels are assayed. Variability in exposure to sertraline between the neonates will be investigated, and the levels will be related to the degree of maladaptation syndrome.

\section{Data management}

Each subject will receive a study number for identification. Data will be collected continuously and entered into the electronic case report file (eCRF) within 2 weeks from collection. The eCRF includes range checks for data values to promote data quality. For the study database, we are using an electronic system, Pheedit version 3.0, provided by Stockholm Healthcare Region, Sweden. The data are encrypted and safely stored on an electronic server at the Karolinska Institute in Stockholm. The e-CRF is previously used in randomised controlled studies. ${ }^{60}$ After completed data collection, the investigators will receive unidentified patient data withdrawn from the CRF and synchronised with data from the internet psychiatry platform. The internet psychiatry platform was also used in our previous study. ${ }^{24}$ The investigators will analyse these data for the different endpoints of the study. The samples from whole blood (mainly to extract DNA for pharmacogenetic analyses in the mother and in the newborns), plasma, cord blood, placenta biopsies and the buccal swabs will be stored in the biobank at Karolinska Institutet in Stockholm, available for analyses and future research.

\section{Statistical methods}

The main analyses will be done according to the intentionto-treat principle. Data will also be analysed per protocol to achieve an efficacy analysis. The primary endpoint, results of BSID III will be analysed with Student's t-test. According to the hypothesis for the primary objective, a superiority analysis will be carried out. In case of no difference in BSID-scores, non-inferiority analyses with a tolerance level of 7 points in the BSID III will be used. We may also include a more conservative sensitivity analysis 
using a tolerance level of 5 points. Multiple imputation will be used for missing data. The variables used in the imputation mode will be smoking, education level, gestational age and parity. Preterm children will be included in the analysis as the chosen psychological and neurological tests are performed at corrected age. All patients' demographic data will be analysed by descriptive (mean, median and range) statistics. Fisher's exact test will be used for dichotomous variables such as infant sex, mother's smoking and optimal/suboptimal neurology at Hammersmith neonatal neurological examination. Throughout the analysis, the mean and SD will be calculated for all continuous data using Student's t-test. The non-normally distributed continuous variables, including the results from Hammersmith neurological scales will be analysed with the Mann-Whitney $\mathrm{U}$ test and the results of MADRS-S by Cohen's effect size. For categorical variables, absolute frequency, percentages and/or proportions are calculated and Fisher's exact test will be used. The treatment effect analysis will be a multilevel model for longitudinal data with timepoints nested within individuals (also known as growth curve modelling) using MADRS-S scores from baseline, each of the 12 weeks in treatment and post-treatment. A $p$ value of $<0.05$ will be considered significant. Subgroup analyses will be performed for prematurity, smokers, parity, maternal age and education level. Spearman's correlation test will be used to test the correlation between maternal and infant drug concentration.

\section{Patient and public involvement}

No patients or public have been involved in the design, recruitment or conduction of the study.

\section{ETHICS AND DISSEMINATION}

\section{Ethics approval and consent to participate}

The Swedish Medical Products Agency (SMPA) approved this study with the approval number 5.1-2016-51237 with the last amendment approved 20160701. The included online supplementary appendix to this article includes a few clarifications in the protocol and is submitted to the Regional Ethical Review Board and the SMPA to be approved as an amendment. Written consent is obtained from all participants at inclusion. At the end of the pregnancy, written consent is obtained from the child's other guardian. All protocol amendments are sent to the Regional Ethical Review Board and the Medical Products Agency for approval before taken in practice. A copy is also sent to Clinicaltrials.gov.

\section{Adverse events (AEs)}

Sertraline is one of the recommended drugs for depression during pregnancy in Stockholm Healthcare Region by the Drug and Therapeutics Committee with high adherence. ${ }^{44}$ Sertraline is also one of the most prescribed SSRIs in the Nordic countries and is widely used across Europe for treatment of depression during pregnancy. ${ }^{3} 4647$ Thereby the therapeutic profile, the adverse effects and the adverse reactions are well known to the study psychiatrists.

An $\mathrm{AE}$ is defined as any undesirable experience associated with the use of the trial drug, sertraline or placebo. A serious adverse event (SAE) is defined as death, a life-threatening event, hospitalisation (initial or prolonged), disability, permanent damage or a congenital birth defect. Expected serious adverse reactions are listed in the Summary of Product Characteristics (SPC). A Suspected Unexpected Serious Adverse Reaction is defined as an AE not listed in the SPC and not mentioned in the SPC as anticipated due to pharmacokinetic properties of the drug, or as a reaction that has occurred with other drugs in this class, but not with the study drug. Unexpected AEs to ICBT will also be reported. All AEs occurring after enrolment are documented in the eCRF. The AEs will be reported to the principal investigator, who will report to the sponsor, and in due cases the KTA for registration in EduraVigilance. All unexpected serious adverse reactions have to be reported within 24 hours after notification by the study personnel. In case of a SAE such as death or a life-threatening event, the primary investigator (PI) will raise the issue of breaking the code and termination of the study with the Data Monitoring and Safety Committee (see below). The committee are responsible for the final decision of study termination. The sponsor will file a yearly Development Safety Update Report (DSUR) to Swedish Medical Products Agency (SMPA).

\section{Monitoring and study safety}

An independent monitor from KTA, Karolinska University Hospital, will continuously control that the study follows approved protocol, with regular audits with the study midwife and the PI. The staff at KTA have all relevant certifications for Good Clinical Practice procedures. Participation in the study is voluntary and women are free to discontinue their participation at any time and for whatever reason without explanations. Midwives at the outpatient clinics and at the delivery clinic have been made aware of this. A Data Monitoring and Safety Board (DSMB) with senior experts in biostatistics, psychiatry, clinical pharmacology (subspecialised in paediatrics) and neonatology. The committee can at any time break the code and undertake necessary precautions including stopping the study if there is any suspicion of an SAE. The committee is independent from the sponsor with no competing interests or involvement in the design, planning or management of the study. The DMSB is also responsible for developing guidelines for interruption of the study and for any interim analyses. The DMC is responsible for deciding if substudy exploratory investigations can be carried out before the recruitment of all 200 mothers, and all 2year neurodevelopment investigations are completed. 


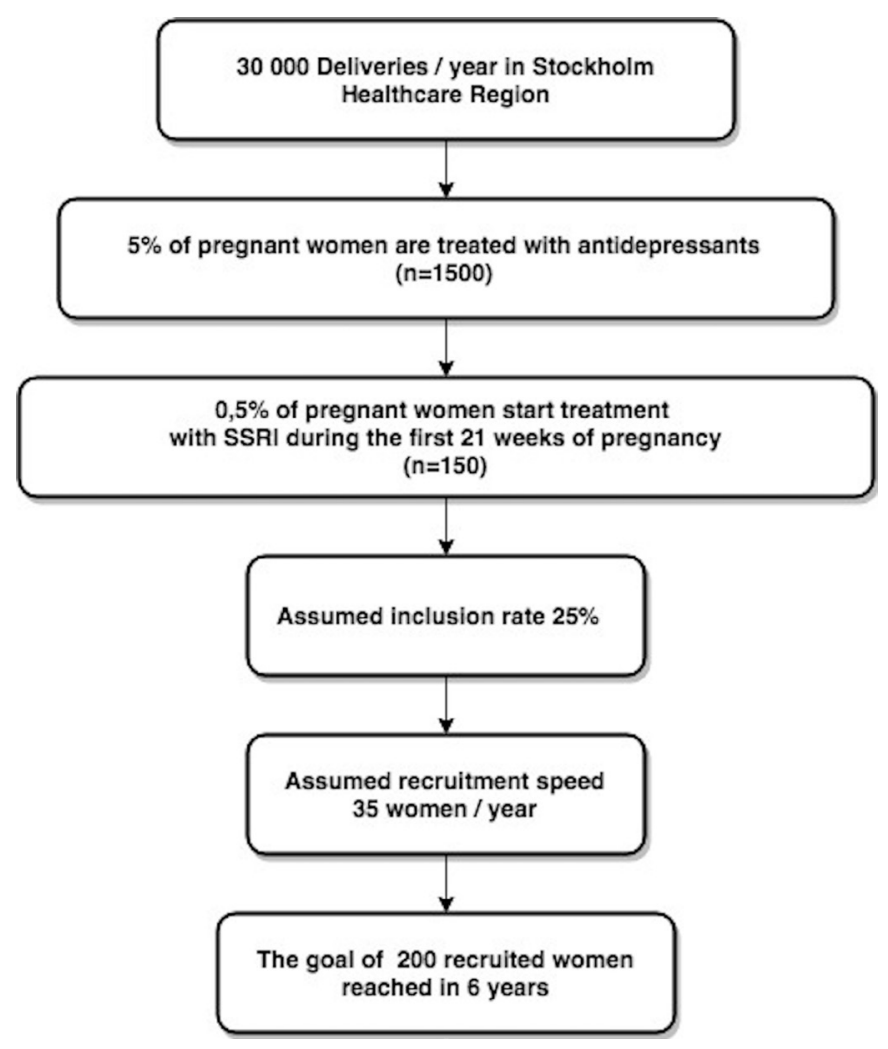

Figure 2 The recruitment base in Stockholm Healthcare Region. Numbers based on an analysis of prescription of SSRIs in pregnant women in years 2013-2016 in the Stockholm Healthcare Region with 2.3 million inhabitants. See acknowledgements. SSRIs, selective serotonin reuptake inhibitors.

\section{Dissemination plan}

This study has as main aim to clarify if any long-term effect on neurodevelopment can be detected in children exposed to sertraline therapy in utero. Other issues will be studied as secondary and tertiary objectives including evaluation of the add-on effect of sertraline to ICBT in moderately depressed pregnant women. The study team will design and implement a knowledge translation (KT) plan using established methods for dissemination of scientific results and modern communication strategies using social media. The KT plan will benefit from strength of being a multidisciplinary scientific group with access to numerous regional, national and international scientific and clinical communities and active involvement of many of the study group scientists in communication with patient groups on psychiatric health and drug therapy during pregnancy. The team has combined years of experience to communicate with colleagues on drug recommendations and achieve high adherence to them using a combination of multifaceted (marketing, continuous medical education, decision support and quality network with colleagues). ${ }^{44} 45$ In summary, we will use established scientific channels to communicate our findings (A): (1) at national and international scientific meetings, (2) as international scientific publications including publishing our study protocol and (3) by contacting relevant international bodies for development of guidelines on best practice in treatment of psychiatric diseases during pregnancy including WHO. We will also use modern communication methods ${ }^{45}$ (B): (1) to summarise our findings for laymen and pregnant groups in Swedish and English and (2) to cooperate with these groups to have information disseminated through their publications and websites. Our aim is to improve knowledge about the need to a higher degree treat depression during pregnancy with the alternatives that we and others have found effective. If we, as hypothesised, will find no effect of sertraline therapy on neurodevelopment in 2-year-old infants, it will be one of the most relevant findings to communicate scientifically and to laymen. The legitimacy of our findings will be high if the results are published in peer-reviewed journals and presented at national and international scientific conferences for scrutiny and discussion.

\section{DISCUSSION}

We hypothesise that sertraline exposure in therapeutic dosage during pregnancy can cause a recognisable effect on the cognition in the offspring as assessed by BSID-III at 2 years of age. A balanced review supports this hypothesis. ${ }^{11}$ Recent large register-based studies ${ }^{16} 17$ imply the importance of a randomised controlled trial to receive sound evidence in this question. As the previous observational studies comment, ${ }^{12} 13$ they have not found a way to adequately adjust for unmeasurable confounders, such as genetic inheritance and disease severity, that we suspect play a significant role when interpreting the results. Using a randomised design in the MAGDALENA study, we minimise the risk for confounding factors.

We also aim to clarify whether combination therapy with ICBT and sertraline is an adequate treatment alternative for moderate depression during pregnancy. This new treatment option provides the pregnant women relevant pregnancy-related questions and the possibility to individually schedule the treatment sessions. Also to our knowledge, no prior placebo-controlled clinical trials have assessed whether combination treatment is superior to treatment with ICBT alone, which we are doing. The risks for the participating women and their offspring are not increased compared with the regular treatment available, as sertraline is widely used in clinical routine in Sweden today. On the contrary, the participating women will have a more thorough follow-up than in clinical practice.

The ethics of performing an RCT in this field has been discussed ${ }^{61}{ }^{62}$ Our study design with ICBT and sertralin or placebo for depression during pregnancy was enabled by recent studies that confirmed the safety and efficacy of ICBT in treating depression, including prenatal depression. ${ }^{2428}$ To date, there is to our knowledge no other study group performing a similar study with the neurodevelopment of the offspring as the main outcome measure. An ongoing Dutch study group randomise clinically undepressed women on antidepressive treatment to either 
guided drug discontinuation with CBT or continuation of drug therapy. ${ }^{63}$ Their primary outcome is the incidence of relapse or recurrence of maternal depression, but they also look at the long-term neurodevelopment with questionnaire based follow-up at 18 months. We consider our method with psychological assessment at 2 years of age more reliable than parental questionnaires. We think that these two studies will complement each other well. Our study will have the unique opportunity to distinguish the effects of exposure to SSRI in utero from the ones of underlying depression, considering our inclusion criteria of moderately depressed women.

A limitation with our study design is the strict inclusion and exclusion criteria potentially causing problems in generalising the results to larger groups of depressed pregnant women. So far, we have not seen any tendencies to exclusion of relevant subgroups. The most common reason for exclusion so far has been not having a severe enough, treatment requiring depression. We collect baseline data from all excluded patients to stay informed about potential statistical biases caused by exclusion. We also know that the recruitment will be challenging, considering the limited study population of clinically depressed, pregnant women without medical treatment with a short time frame for recruitment. We have addressed this using a combined approach recruiting both at ordinary antenatal clinics and through social media, pregnancy applications on smartphones as well as with marketing campaigns. Studies worldwide show that three out of four pregnant women use a pregnancy-related application, showing their great potential. $^{39}{ }^{40}$ Our web-based recruitment and treatment also gives us the possibility to invite additional study centres across the country if necessary to complete the recruitment. We are constantly facing fears from potential subjects of taking antidepressant treatment during pregnancy.

We have been working with this study for 4 years, specifying and completing the protocol and getting ethical approval and permission from the national Medical Products Agency. In addition, it has taken an additional year to test the feasibility by completing the first five patients through the pregnancy and delivery parts of the trial (January 2018). During these 5 years, the multidisciplinary research group with less and more experienced members has had regular meetings. So far, we have not met any problems in raising needed funds, received both from major national and regional funders of clinical research as well as from multiple funders specialised to support studies on safe care of mother and child during pregnancy and in early life. Therefore, we are confident to finish the recruitment within 6years, based on our calculations on data from Stockholm Healthcare Region (figure 2) and publish the results in another 3 years.

\footnotetext{
Author affiliations

${ }^{1}$ Division of Paediatrics, Department of Clinical Science, Intervention and Technology (CLINTEC), Karolinska Institutet, Huddinge, Stockholm

${ }^{2}$ Department of Paediatrics, Karolinska University Hospital, Stockholm, Sweden

${ }^{3}$ Division of Obstetrics and Gynaecology, Department of Clinical Science,

Intervention and Technology (CLINTEC), Karolinska Institutet, Stockholm, Sweden
}

${ }^{4}$ Department of Obstetrics and Gynaecology, Karolinska University Hospital, Stockholm, Sweden

${ }^{5}$ Centre for Psychiatry Research, Department of Clinical Neuroscience, Karolinska Institutet, Stockholm, Sweden

${ }^{6}$ Psychiatry South West, Stockholm Health Care Region, Stockholm, Sweden ${ }^{7}$ Department of Psychology, Faculty of Health and Life Sciences, Linnaeus University, Stockholm, Växjö, Sweden

${ }^{8}$ Department of Women's and Children's Health, Karolinska Institutet, Stockholm, Sweden

${ }^{9}$ Division of Clinical Pharmacology, Department of Laboratory Medicine, Karolinska Institutet, Stockholm, Sweden

${ }^{10}$ Department of Clinical Pharmacology, Karolinska University Hospital, Stockholm, Sweden

Acknowledgements We used the SPIRIT checklist when writing this article. We would like to acknowledge M Juhasz-Haverinen at Public Healthcare Services Committee (HSF) at Stockholm Healthcare Region (SLL) for providing data on our recruitment base of patients for the study (figure 2) as well as M Bendix, M L Dahl and C Ruck for input on the study protocol at an early phase. We are grateful for statistical advice provided at various stages in the design of the study provided by the biostatistician Ulf Hammar at Karolinska Institutet. We would also like to acknowledge all women, their babies and their families who participate in the MAGDALENA study and the numerous midwives, obstetricians and staff at all MAGDALENA recruiting Antenatal Clinics and Maternity Wards and Delivery Units in Stockholm Healthcare Region.

Contributors EH and LLG wrote the initial study protocol article with the help of BS-VS, LF and AF. KW is the principal investigator and JN, LLG, MB and VK are members of the governing study board. $M B$ is the sponsor and the main applicant and receiver of our initial groundbreaking funding decision from the Swedish Research Council. MB is also a senior researcher with full responsibility of the study design and participation in completion of the manuscript. JN, KW and MB conceived the study protocol, and KW wrote the initial draft of the study protocol. EA is developing an exploratory study protocol studying attachment in a subgroup of the pregnant women. MB, SF and EH launched the open recruitment through different social media platforms. VK and EF adapted the ICBT procedure for use in our pregnant women and are responsible for the ICBT treatment. They also directed the pilot study. AF is the study midwife coordinating the visits and the day-to-day management of the study. MB-W, MS and AG are the study psychiatrists responsible for the initial assessment and inclusion in the study as well as for the clinical decisions about pharmacotherapy. EMN is the psychiatry nurse responsible for the treatment evaluations. All authors have in different ways been involved in different degrees in the development of study design and take responsibility for the final study design. All authors have contributed to the protocol development and have read and approved the final protocol that we now use after revisions as well as this manuscript. EH and LLG coordinated the completion of the study protocol article.

Funding The study was funded by Vetenskapsrådet (521-2012-3466), Stockholms Läns Landsting (533069, 571301), Lilla Barnets Fond.

Competing interests None declared.

Patient consent Not required.

Ethics approval The Regional Ethical Review Board at Karolinska Institutet in Stockholm approved this study with the approval number 2014/952-31 with the last amendment approved 20180507.

Provenance and peer review Not commissioned; externally peer reviewed.

Open access This is an open access article distributed in accordance with the Creative Commons Attribution Non Commercial (CC BY-NC 4.0) license, which permits others to distribute, remix, adapt, build upon this work non-commercially, and license their derivative works on different terms, provided the original work is properly cited, appropriate credit is given, any changes made indicated, and the use is non-commercial. See: http:// creativecommons.org/licenses/by-nc/4.0/.

\section{REFERENCES}

1. Le Strat Y, Dubertret C, Le Foll B. Prevalence and correlates of major depressive episode in pregnant and postpartum women in the United States. J Affect Disord 2011;135(1-3):128-38. 
2. Gavin NI, Gaynes BN, Lohr KN, et al. Perinatal depression: a systematic review of prevalence and incidence. Obstet Gynecol 2005;106(5 Pt 1):1071-83.

3. Zoega $\mathrm{H}$, Kieler $\mathrm{H}$, Nørgaard $\mathrm{M}$, et al. Use of SSRI and SNRI Antidepressants during Pregnancy: A Population-Based Study from Denmark, Iceland, Norway and Sweden. PLoS One 2015;10:e0144474.

4. Qiu C, Williams MA, Calderon-Margalit R, et al. Preeclampsia risk in relation to maternal mood and anxiety disorders diagnosed before or during early pregnancy. Am J Hypertens 2009;22:397-402.

5. Grote NK, Bridge JA, Gavin AR, et al. A meta-analysis of depression during pregnancy and the risk of preterm birth, low birth weight, and intrauterine growth restriction. Arch Gen Psychiatry 2010;67:1012-24.

6. Field T, Diego M, Hernandez-Reif M. Prenatal depression effects on the fetus and newborn: a review. Infant Behav Dev 2006;29:445-55.

7. Gentile S. Untreated depression during pregnancy: Short- and long-term effects in offspring. A systematic review. Neuroscience 2017;342:154-66.

8. Nörby U, Forsberg L, Wide K, et al. Neonatal Morbidity After Maternal Use of Antidepressant Drugs During Pregnancy. Pediatrics 2016;138:e20160181.

9. Andrade SE, Reichman ME, Mott K, et al. Use of selective serotonin reuptake inhibitors (SSRIs) in women delivering liveborn infants and other women of child-bearing age within the U.S. Food and Drug Administration's Mini-Sentinel program. Arch Womens Ment Health 2016;19:969-77.

10. Furu K, Kieler H, Haglund B, et al. Selective serotonin reuptake inhibitors and venlafaxine in early pregnancy and risk of birth defects: population based cohort study and sibling design. BMJ 2015;350:h1798.

11. Oberlander TF, Gingrich JA, Ansorge MS. Sustained neurobehavioral effects of exposure to SSRI antidepressants during development: molecular to clinical evidence. Clin Pharmacol Ther 2009;86:672-7.

12. Rai D, Lee BK, Dalman C, et al. Parental depression, maternal antidepressant use during pregnancy, and risk of autism spectrum disorders: population based case-control study. BMJ 2013;346:f2059.

13. Rai D, Lee BK, Dalman C, et al. Antidepressants during pregnancy and autism in offspring: population based cohort study. BMJ 2017;358:|2811.

14. Croen LA, Grether JK, Yoshida CK, et al. Antidepressant use during pregnancy and childhood autism spectrum disorders. Arch Gen Psychiatry 2011;68:1104-12.

15. El Marroun $\mathrm{H}$, White TJ, van der Knaap NJ, et al. Prenatal exposure to selective serotonin reuptake inhibitors and social responsiveness symptoms of autism: population-based study of young children. $\mathrm{Br} \mathrm{J}$ Psychiatry 2014;205:95-102.

16. Brown HK, Ray JG, Wilton AS, et al. Association Between Serotonergic Antidepressant Use During Pregnancy and Autism Spectrum Disorder in Children. JAMA 2017;317:1544-52.

17. Sujan AC, Rickert ME, Öberg AS, et al. Associations of Maternal Antidepressant Use During the First Trimester of Pregnancy With Preterm Birth, Small for Gestational Age, Autism Spectrum Disorder, and Attention-Deficit/Hyperactivity Disorder in Offspring. JAMA 2017;317:1553-62.

18. Morales DR, Slattery J, Evans S, et al. Antidepressant use during pregnancy and risk of autism spectrum disorder and attention deficit hyperactivity disorder: systematic review of observational studies and methodological considerations. BMC Med 2018 (6(1):6 (published Online First: 2018/01/16).

19. Hedman E, Ljótsson B, Kaldo V, et al. Effectiveness of Internet-based cognitive behaviour therapy for depression in routine psychiatric care. J Affect Disord 2014;155:49-58.

20. O'Mahen HA, Woodford J, McGinley J, et al. Internet-based behavioral activation--treatment for postnatal depression (Netmums): a randomized controlled trial. J Affect Disord 2013;150:814-22.

21. Danaher BG, Milgrom J, Seeley JR, et al. MomMoodBooster WebBased Intervention for Postpartum Depression: Feasibility Trial Results. J Med Internet Res 2013;15:e242.

22. O'Mahen HA, Richards DA, Woodford J, et al. Netmums: a phase II randomized controlled trial of a guided Internet behavioural activation treatment for postpartum depression. Psychol Med 2014;44:1675-89.

23. Pugh NE, Hadjistavropoulos HD, Dirkse D. A Randomised Controlled Trial of Therapist-Assisted, Internet-Delivered Cognitive Behavior Therapy for Women with Maternal Depression. PLoS One 2016;11:e0149186.

24. Forsell E, Bendix M, Holländare F, et al. Internet delivered cognitive behavior therapy for antenatal depression: A randomised controlled trial. J Affect Disord 2017;221:56-64.
25. Nationella riktlinjer för vård vid depression och ångestsyndrom: Socialstyrelsen. 2017. https://roi.socialstyrelsen.se/riktlinjer/ nationella-riktlinjer-for-vard-vid-depression-och-angestsyndrom (accessed 16 Feb 2018).

26. Cuijpers P, Sijbrandij M, Koole SL, et al. Adding psychotherapy to antidepressant medication in depression and anxiety disorders: a meta-analysis. World Psychiatry 2014;13:56-67.

27. Andrews G, Cuijpers P, Craske MG, et al. Computer therapy for the anxiety and depressive disorders is effective, acceptable and practical health care: a meta-analysis. PLoS One 2010;5:e13196.

28. Cuijpers P, Donker T, van Straten A, et al. Is guided self-help as effective as face-to-face psychotherapy for depression and anxiety disorders? A systematic review and meta-analysis of comparative outcome studies. Psychol Med 2010;40:1943-57.

29. Lindqvist PG, Nasiell J, Gustafsson LL, et al. Selective serotonin reuptake inhibitor use during pregnancy increases the risk of postpartum hemorrhage and anemia: a hospital-based cohort study. $J$ Thromb Haemost 2014;12:1986-92.

30. Moses-Kolko EL, Bogen D, Perel J, et al. Neonatal signs after late in utero exposure to serotonin reuptake inhibitors: literature review and implications for clinical applications. JAMA 2005;293:2372-83.

31. Basterzi AD, Aydemir Çigdem, Kisa C, et al. IL-6 levels decrease with SSRI treatment in patients with major depression. Hum Psychopharmacol 2005;20:473-6.

32. Park Y-M. Serum Prolactin Levels in Patients with Major Depressive Disorder Receiving Selective Serotonin-Reuptake Inhibitor Monotherapy for 3 Months: A Prospective Study. Psychiatry Investig 2017;14:368-71.

33. Susser LC, Sansone SA, Hermann AD. Selective serotonin reuptake inhibitors for depression in pregnancy. Am J Obstet Gynecol 2016;215:722-30.

34. Nonnenmacher N, Noe D, Ehrenthal JC, et al. Postpartum bonding: the impact of maternal depression and adult attachment style. Arch Womens Ment Health 2016;19:927-35.

35. Freeman MP, Nolan PE, Davis MF, et al. Pharmacokinetics of sertraline across pregnancy and postpartum. $J$ Clin Psychopharmacol 2008;28:646-53.

36. Helldén A, Madadi P. Pregnancy and pharmacogenomics in the context of drug metabolism and response. Pharmacogenomics 2013;14:1779-91.

37. Saiz-Rodríguez M, Belmonte C, Román M, et al. Effect of Polymorphisms on the Pharmacokinetics, Pharmacodynamics and Safety of Sertraline in Healthy Volunteers. Basic Clin Pharmacol Toxicol.

38. Viuff A-CF, Pedersen LH, Kyng K, et al. Antidepressant medication during pregnancy and epigenetic changes in umbilical cord blood: a systematic review. Clin Epigenetics 2016;8:94.

39. Lupton D, Pedersen S. An Australian survey of women's use of pregnancy and parenting apps. Women and Birth 2016;29:368-75.

40. Osma J, Barrera AZ, Ramphos E. Are Pregnant and Postpartum Women Interested in Health-Related Apps? Implications for the Prevention of Perinatal Depression. Cyberpsychol Behav Soc Netw 2016;19:412-5.

41. Rubertsson C, Börjesson $\mathrm{K}$, Berglund $\mathrm{A}$, et al. The Swedish validation of Edinburgh Postnatal Depression Scale (EPDS) during pregnancy. Nord J Psychiatry 2011;65:414-8.

42. First MB. Structured Clinical Interview for DSM-IV Axis I Disorders, Clinician Version (SCID-CV). Spitzer C, ed. Washington, D.C. American Psychiatric Press, Inc, 1996.

43. SLL. Regionalt vårdprogram psykisk sjukdom i samband med graviditet och spädbarnsperiod. pp. 20 \& 34 (Translated: Stockholm City Council - Regional recommendations of treatment of psychiatric disease during pregnancy and infancy. 2014. http://www1. psykiatristod.se/Global/Psykiatristod/Bilagor/RVP_GravDepp_webb. pdf (accessed 31 May 2018).

44. Gustafsson LL, Wettermark B, Godman B, et al. The 'Wise List'- A Comprehensive Concept to Select, Communicate and Achieve Adherence to Recommendations of Essential Drugs in Ambulatory Care in Stockholm. Basic Clin Pharmacol Toxicol 2011;108:224-33.

45. Eriksen J, Gustafsson LL, Ateva K, et al. High adherence to the 'Wise List' treatment recommendations in Stockholm: a 15-year retrospective review of a multifaceted approach promoting rational use of medicines. BMJ Open 2017;7:e014345.

46. Charlton RA, Jordan S, Pierini A, et al. Selective serotonin reuptake inhibitor prescribing before, during and after pregnancy: a population-based study in six European regions. BJOG 2015;122:1010-20.

47. Cleare $\mathrm{A}$, Pariante $\mathrm{CM}$, Young $\mathrm{AH}$, et al. Evidence-based guidelines for treating depressive disorders with antidepressants: A revision of the 2008 British Association for Psychopharmacology guidelines. $J$ Psychopharmacol 2015;29:459-525. 
48. Albers CA, Grieve AJ. Test Review: Bayley, N. (2006). Bayley Scales of Infant and Toddler Development- Third Edition. San Antonio, TX: Harcourt Assessment. J Psychoeduc Assess 2007;25:180-90.

49. Bode MM, D'Eugenio DB, Mettelman BB, et al. Predictive validity of the Bayley, Third Edition at 2 years for intelligence quotient at 4 years in preterm infants. J Dev Behav Pediatr 2014;35:570-5.

50. Serenius F, Källén K, Blennow M, et al. Neurodevelopmental outcome in extremely preterm infants at 2.5 years after active perinatal care in Sweden. JAMA 2013;309:1810-20.

51. Santucci AK, Singer LT, Wisniewski SR, et al. Impact of prenatal exposure to serotonin reuptake inhibitors or maternal major depressive disorder on infant developmental outcomes. J Clin Psychiatry 2014;75:1088-95.

52. Casper RC, Fleisher BE, Lee-Ancajas JC, et al. Follow-up of children of depressed mothers exposed or not exposed to antidepressant drugs during pregnancy. J Pediatr 2003;142:402-8.

53. Cardonick EH, Gringlas MB, Hunter K, et al. Development of children born to mothers with cancer during pregnancy: comparing in utero chemotherapy-exposed children with nonexposed controls. Am J Obstet Gynecol 2015;212:658.e1-658.e8.

54. Wouldes TA, LaGasse LL, Huestis MA, et al. Prenatal methamphetamine exposure and neurodevelopmental outcomes in children from 1 to 3years. Neurotoxicol Teratol 2014;42:77-84.

55. Tamayo-Sarver JH, Dawson NV, Hinze SW, et al. The effect of race/ethnicity and desirable social characteristics on physicians' decisions to prescribe opioid analgesics. Acad Emerg Med 2003;10:1239-48.

56. Svanborg P, Åsberg M. A new self-rating scale for depression and anxiety states based on the Comprehensive Psychopathological Rating Scale. Acta Psychiatr Scand 1994;89:21-8.
57. Dubowitz L, Ricciw D, Mercuri E. The Dubowitz neurological examination of the full-term newborn. Ment Retard Dev Disabil Res Rev 2005;11:52-60.

58. Forsberg L, Navér L, Gustafsson LL, et al. Neonatal adaptation in infants prenatally exposed to antidepressants--clinical monitoring using Neonatal Abstinence Score. PLoS One 2014;9:e111327.

59. Friedman DD, Beebe B, Jaffe J, et al. Microanalysis of 4-Month Infant Vocal Affect Qualities and Maternal Postpartum Depression. Clin Soc Work J 2010;38:8-16.

60. Foukakis T, von Minckwitz G, Bengtsson N-O, et al. Effect of Tailored Dose-Dense Chemotherapy vs Standard 3-Weekly Adjuvant Chemotherapy on Recurrence-Free Survival Among Women With High-Risk Early Breast Cancer. JAMA 2016;316:1888-96.

61. Oberlander TF, Vigod SN. Developmental Effects of Prenatal Selective Serotonin Reuptake Inhibitor Exposure in Perspective: Are We Comparing Apples to Apples? J Am Acad Child Adolesc Psychiatry 2016;55:351-2.

62. Coverdale JH, McCullough LB, Chervenak FA. The ethics of randomized placebo-controlled trials of antidepressants with pregnant women: a systematic review. Obstet Gynecol 2008;112:1361-8.

63. Molenaar NM, Brouwer ME, Bockting CLH, et al. Stop or go? Preventive cognitive therapy with guided tapering of antidepressants during pregnancy: study protocol of a pragmatic multicentre noninferiority randomized controlled trial. BMC Psychiatry 2016;16:72.

64. Montgomery SA, Asberg M. A new depression scale designed to be sensitive to change. Br J Psychiatry 1979;134:382-9.

65. Romeo DM, Cioni M, Palermo F, et al. Neurological assessment in infants discharged from a neonatal intensive care unit. Eur J Paediatr Neurol 2013;17:192-8. 